\title{
Resilience of operated tunnels under extreme surcharge: field study
}

\author{
Hongwei Huang ${ }^{\text {i) }}$ and Dongming Zhang ${ }^{\text {ii) }}$ \\ i) Professor, Email: huanghw@tongji.edu.cn, Dept. of Geotechnical Engineering, Tongji University, 1239 Siping Rd, Shanghai, 200092, \\ China. \\ ii) Ph.D Student, Email: 09zhang@tongji.edu.cn, Ditto.
}

\begin{abstract}
To date, Shanghai has built an intensive network of metro tunnel system. In the operation of such a complex tunnel system, the vulnerability and the recovery of the segmental linings subjected to unexpected disruptions are badly concerned by the engineers and owners. In this respect, the analysis of segmental lining resilience, i.e., the ability to absorb the disruption caused by the hazards and then the ability to recover to the acceptable level of the functionality, is necessary for the safety of the tunnels. In Shanghai, from time to time, some dumped soils from construction site elsewhere are found to be disposed without permission at the ground surface above the operated tunnels. When the volume of these dumped soils are significantly large, e.g., a height of $5-8 \mathrm{~m}$, it will inevitably do threats to the safety of the underlying operated tunnels. In this paper, the resilience of the segmental lining system subjected to this unexpected extreme surcharge loads will be characterized with a real field case in Shanghai. The convergence deformation is regarded as the functionality of tunnels. The functionality curve is described using the measured data from a detailed monitoring program. The unloading of the dumped soils above the surface and the grouting around tunnel from surface are the two recovery measures both implemented in this field case. The calculated metric of resilience indicates that the functionality of the tunnels can be bounced back to a certain level by these two recovery measures. Besides, the rapidity of the recovery of these two measures is compared. Finally, some discussion and concluding remarks on the resilience of operated tunnels subjected to this extreme surcharge load are presented.
\end{abstract}

Keywords: resilience, segmental linings, hazards, field study

\section{INTRODUCTION}

The metro system in metropolis is one of the best solutions to relieve the congested transportation pressures. In Shanghai, the metro system with a $538 \mathrm{~km}$ of mileage takes up $43 \%$ of daily public transportation volume. In operating such a huge system, the resilience of tunnel structures subjected to any unexpected hazards is badly concerned by the government and the engineers. Unfortunately, some human-related hazard, from time to time, is found to act on the tunnels. Fig. 1 shows a pie chart for these recorded human-related hazards from 2004 to 2013, statistics from the personal communication with engineers from Shanghai Metro Group Co., Ltd. It is clear that the extreme surcharge, caused by the dumped soils loaded on the ground surface without permission, is the most significant type of human hazard.

The robustness of the tunnel response to the extreme surcharge and the recovery from such a disaster are the key aspects for a lifetime performance assessment. These two aspects compose the basic concept of a system resilience, which is receiving increasing attentions these days (Francis and Bekera, 2014).
However, the study on the system resilience still remains at the stage of conceptualization so far (Karen, 2014). In view of this situation, this paper presented a detailed assessment of the tunnel lining resilience subjected to the extreme surcharge loading on ground surface by using a field case study. First, a general definition of the tunnel resilience is established. By probing into the field case and monitoring data, the vulnerability and the recovery index is then calculated. The corresponding resilience index is thus obtained using the general metric of the resilience. Finally, a discussion on the tunnel resilience subjected to the extreme surcharge in this case would be provided.

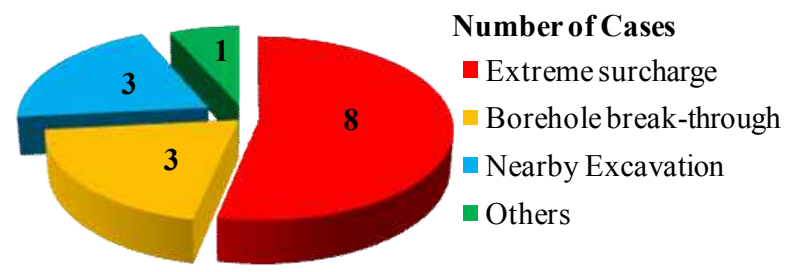

Fig. 1. Statistic of lining disaster in Shanghai from 2004 to 2013 


\section{RESILIENCE DEFINITION}

Before a detailed description of the tunnel resilience, the index for tunnel performance should be first specified. To be an index, it should be easily measured in site and significantly reflecting the structural response. The tunnel horizontal convergence $\Delta \mathrm{D}$ is adopted in this paper (shown in Fig. 2), which is probably the widely used index both in practices (MTPRC, 2004) and researches (Mair, 2008).
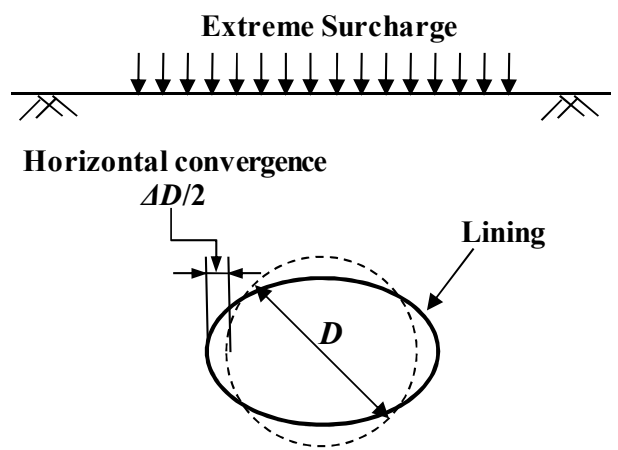

Fig. 2. Performance index of lining convergence

Eq. 1 is denoted as the performance index $Q_{n}(t)$ by a normalization transformation form with $\Delta D$, where $\Delta D_{0}$ is the initial convergence deflection once the tunnel is built and $\Delta D(t)$ is the convergence at time $t$ which can consider the degradation effect with time.

$$
Q_{n}(t)=\frac{\Delta D_{0}}{\Delta D(t)}
$$

The resilience is explained conceptually as the ability of a system to absorb the disruption caused by the hazards and the ability to recover to the acceptable performance level (Ayyub, 2014). Based on the frame proposed by Ayyub (2014), Fig. 3 has illustrated the detailed frame of the lifetime performance evolution for tunnel convergence. From a general perspective, there are three stages including before surcharge, after surcharge and after recovery. The resilience assessment locates in the second stage, i.e., after surcharge.

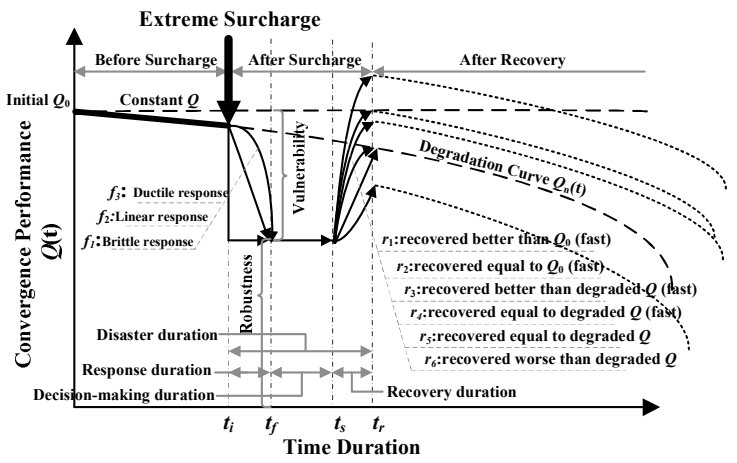

Fig. 3. Definition of convergence resilience for tunnels
Once the surcharge is loaded at time $t_{\mathrm{i}}$, the tunnel convergence will make a response to this action. The performance will then experience a decrease described by function $f(\mathrm{t})$. The residual performance $f_{\mathrm{d}}$ after this response (at time $t_{\mathrm{f}}$ ) stands for the robustness of the tunnel lining. Due to the time cost for decision-making process, the performance will experience a relative stable evolution illustrated by function $s(\mathrm{t})$. Then, once the recovery measures are implemented at time $t_{\mathrm{s}}$, the recovery will take place until the time $t_{\mathrm{r}}$ reaching to an acceptable level of performance. Hence, the resilience metric can be visually explained by the ratio of the area for the performance evolution function, i.e., $f(\mathrm{t}), s(\mathrm{t})$ and $r(\mathrm{t})$, over the area of normal performance function $Q_{n}(\mathrm{t})$. Mathematically, the resilience index is calculated by following equation:

$$
\begin{aligned}
& \operatorname{Re}=\frac{F\left(t_{f}-t_{i}\right)+S\left(t_{s}-t_{f}\right)+R\left(t_{r}-t_{s}\right)}{t_{r}-t_{i}} \\
& F=\int_{t_{t_{f}}}^{t_{f}} f d t / \int_{t_{i}}^{t_{f}} Q_{n} d t \\
& S=\int_{t_{f}}^{t_{s}} s d t / \int_{t_{f}}^{t_{s}} Q_{n} d t \\
& R=\int_{t_{s}}^{t_{r}} r d t / \int_{t_{s}}^{t_{r}} Q_{n} d t
\end{aligned}
$$

Several dimensions can be covered in the above metric of the resilience, including degradation, robustness, vulnerability, rapidity and recovery. Details are summarized in Table 1.

Table 1. Resilience dimension and its property

\begin{tabular}{lcl}
\hline \multicolumn{1}{c}{ Dimension } & Symbol & \multicolumn{1}{c}{ Property } \\
\hline Degradation & $f_{\mathrm{i}}$ & Degraded performance $Q_{n}(\mathrm{t})$ at $t_{\mathrm{i}}$ \\
Robustness & $f_{\mathrm{d}}$ & Residual performance at $t_{\mathrm{f}}$ \\
Vulnerability & $f_{\mathrm{l}}=f_{\mathrm{i}}-f_{\mathrm{d}}$ & Performance loss at $t_{\mathrm{f}}$ \\
Rapidity & $\Delta T=t_{\mathrm{r}}-t_{\mathrm{s}}$ & Speed of recovery \\
Recovery & $f_{\mathrm{r}}$ & Recovered performance \\
\hline
\end{tabular}

\section{INTRODUCTION OF FIELD CASE}

\subsection{Site information}

In Shanghai, there were 8 recorded cases of unexpected extreme surcharge on the ground surface above the operated metro tunnels from 2004 to 2013. One of the recent cases will be introduced here in this paper. The case happened in the metro line 2 in east of Shanghai. The tunnel was excavated by earth pressures balance shield machine. Fig. 4 has shown a longitudinal cross section of the case along the alignment of the affected tunnel. The initial cover depth of the tunnel axis lied between $16 \mathrm{~m}$ and $20 \mathrm{~m}$. The tunnel was mostly driven through the two-layered formation consisting of muddy clay and the clay. The soil information provided by boreholes shown in Fig. 4 indicates that both of the two types of surrounding soils were very sensitive soft clays with a high water content. Due to page limit, the detailed soil information is not presented here. 


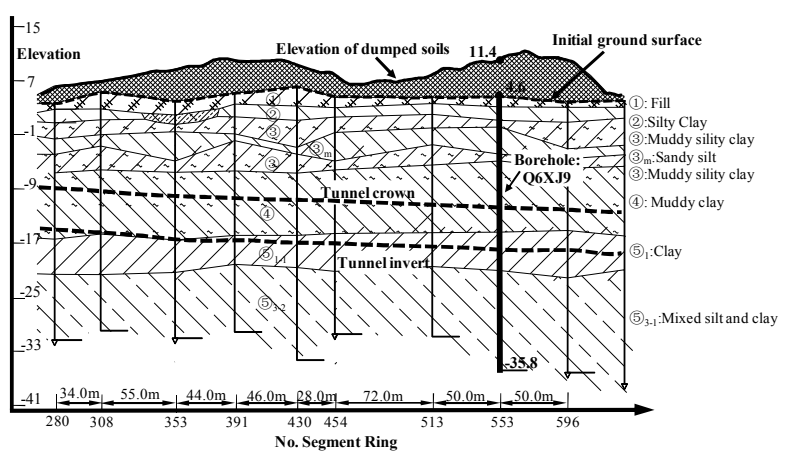

Fig. 4. Cross section of surcharged area along the tunnel

The construction of the tunnel had been completed by Oct of 2008. However, in May of 2010, a metro train driver found a broken segment of concrete lining dropped at the tunnel sidewall, which uncovered this huge potential disaster caused by the extreme surcharge. After an immediate geological investigation, the surcharge was caused by the dumped soils without permission just above the tunnel. The height of dumped soils ranged from 5-8m, shown in Fig. 4. A total of 300 rings, shown in Fig. 4, were affected by the surcharge. Large convergence deflection $(\Delta D=6-22 \mathrm{~cm})$, segment cracks, joint opens and even the failure of steel bolts were detected in these 300 rings.

\subsection{Monitoring}

A detailed structural monitoring program was then implemented for the whole tunnel between two adjacent stations after the discovery of the surcharge. Fig. 5 has illustrated the monitoring program on the tunnel lining. The lining had an out-diameter of $6.2 \mathrm{~m}$ and a thickness of $0.35 \mathrm{~m}$. The lining consisted of six segments, which were connected by the steel bolts, shown in Fig. 5. The horizontal convergence was monitored by using the total stations at the springlines. During the decision making period, the monitoring frequency is once per month, while during the construction of the recovery measures, the frequency increased to once per day.

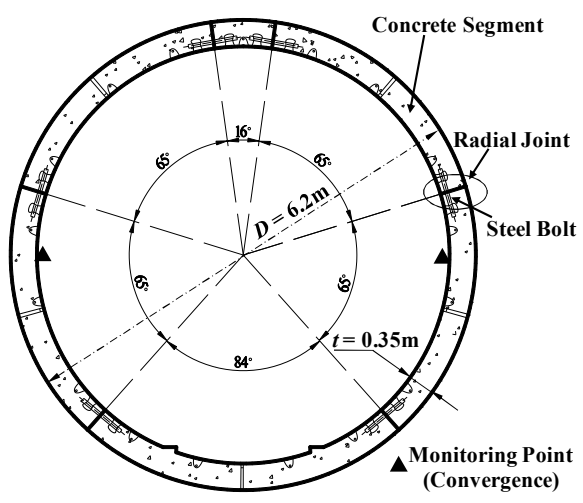

Fig. 5. Section of segmental lining and monitoring program

\subsection{Recovery measures}

Although it was not quite certain, the metro company had inferred the dumping of the soils on the ground surface happened in Dec 2008. After the discovery of the surcharge in May 2010, an immediate action of unloading the soils was made to first recover the tunnel convergence as most as possible. This time period started from May 2010 to Mar 2011. After the unloading of the surcharges, the tunnel convergence was found to be slowly bounced back. The metro company then decided to apply the soil grouting to the soils at two sides of the tunnel to speed up the recovery of tunnel convergence. The second stage of recovery measures started from May 2014 to Jul 2014. The overall timetable of the event on this field case has been illustrated in Fig. 6.

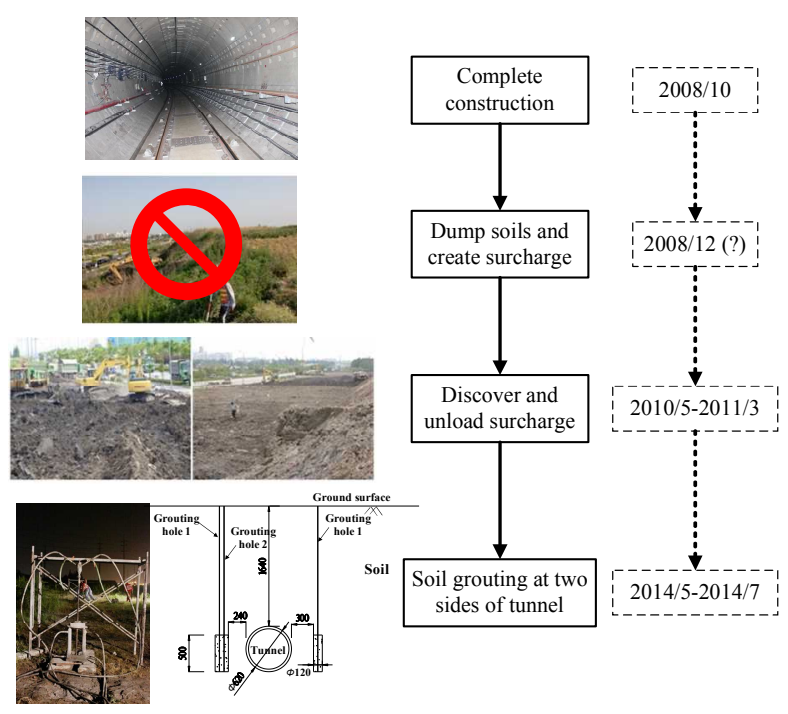

Fig. 6. Timetable of each event on the tunnel

\section{RESILISENCE METRIC}

\subsection{Degradation curve $Q(t)$}

Since the monitoring has been applied along the whole tunnel between two adjacent stations, the normal performance of the lining convergence can be captured by the field data of those unaffected lining rings. Fig. 7 has plotted the data from ring 199, 261 and 272 against the time. Note that the data shown in Fig. 7 is in the form of performance index calculated by using Eq. 1 .

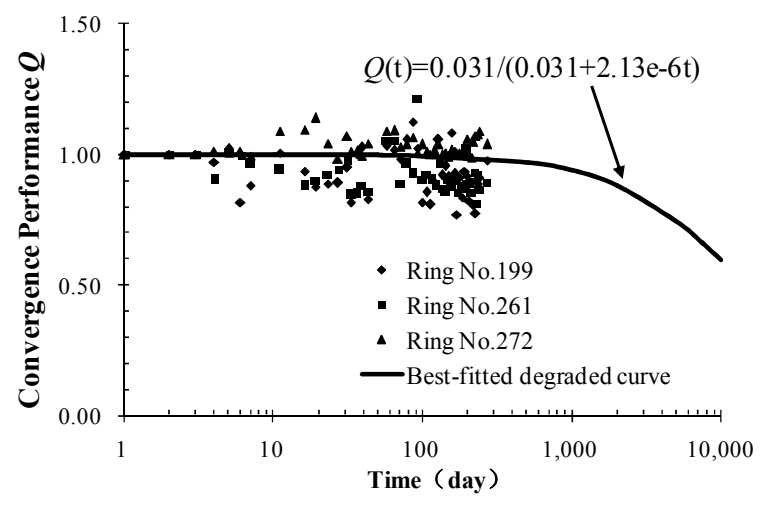

Fig. 7. Degradation curve of convergence performance $Q(\mathrm{t})$

It is not surprising that the field data have shown a large scatter along the time $t$. But by applying the 
moving average technique and curve fitting analysis, a reasonable normal performance evolution function can be obtained as follows:

$$
Q(t)=\frac{\Delta D_{0}}{\Delta D_{0}+q(t)}=\frac{0.031}{0.031+2.13 \times 10^{-6} t}
$$

where $q(\mathrm{t})$ is the increment of the convergence with time $t$ (day). The number for the numerator in Eq. 6 represents the initial convergence of $0.031 \mathrm{~m}$. This evolution function is also plotted in Fig. 7. From Fig. 7, it is inferred that it will take nearly 30 years for degrading $50 \%$ of the initial performance if no enhancement or repair work will be applied.

\subsection{Structure response curve $f(t)$}

Since the occurrence of the surcharge was inferred in Dec 2008 and the discovery of the surcharge was in May 2010, the response period for the tunnel structure lasted almost 550 days. The affected lining ring No. 388 was selected for analysis of resilience. In May 2010 , the convergence $\Delta D$ of ring 388 was $15.2 \mathrm{~cm}$, i.e., $24.5 \%{ }_{0} D$. Fig. 8 has plotted the response curve $f(t)$ against time $t$.

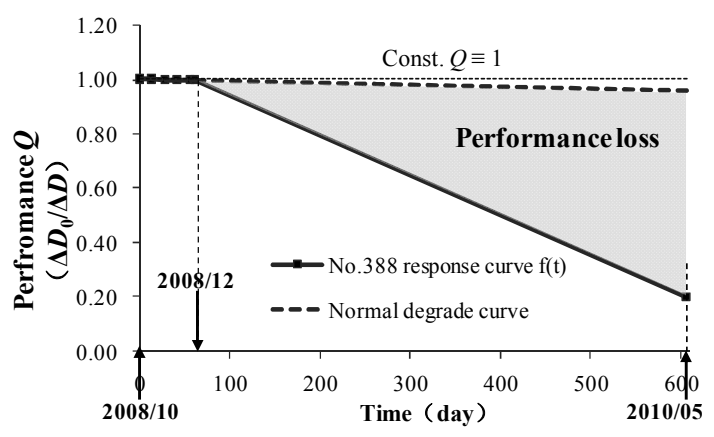

Fig. 8. Robustness of convergence performance subjected to surcharge loading

Since no information during the response period can be obtained, the response curve $f(\mathrm{t})$ is fitted by a linear curve expressed as follows:

$$
f(t)=0.995-0.00144(t-61), 61 \leq t \leq 606
$$

It might be a little bit arbitrary that the response curve follows a linear form. But it has to be confessed that since the field monitoring has not been commenced before the tunnel operation, there is no evidence and information to indicate the lining performance disrupted in some specific day or period. If no surcharge is applied, the normal performance $Q(t)$ at time $t_{\mathrm{f}}$ should be 0.96 . However, due to the loading of extreme surcharge, the residual performance has been reduced to $0.21 .75 \%$ of the performance is lost at the end of the response period.

\subsection{Structure recovery curve $r(t)$}

As shown in Fig. 6, there are two recovery stages, including the unloading and the soil grouting. Fig. 9a and $9 \mathrm{~b}$ have plotted the performance recovery history for the two measures, respectively. The unloading of the surcharge has increased the performance from 0.21 to 0.23 by almost 250 days. This increase experiences two stages with different speeds. The turning point is actually the time that the unloading work above the ring was finished in site. In general, the contribution of the unloading to the recovery performance is limited by $2 \%$. After 3 years, the metro company decided to speed up the recovery by using the soil grouting measures. It is clear from Fig. 9b that the rapidity of this recovery stage is quite significant. The recovered performance has reached to 0.34 . In addition, the increase from 0.23 to 0.34 only takes 50 days.
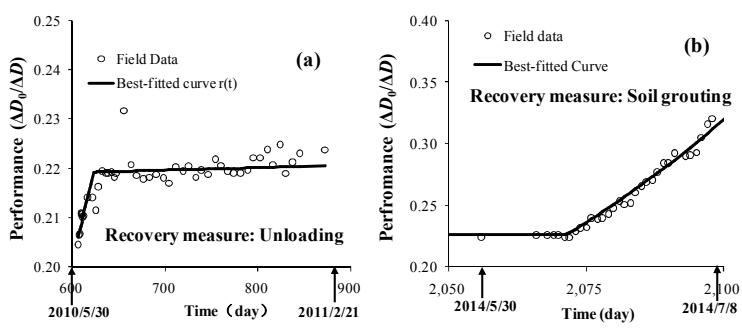

Fig. 9. Performance recovery due to specific measures: a): unloading and $b$ ): grouting

As shown in Fig. 9, the recovery data at the first stage can be fitted by a bio-linear curve and the data at the second stage can be fitted by an exponential curve. Mathematically, the recovery curves can be represented by the following equation:

$$
r(t)=\left\{\begin{array}{l}
7.68 \times 10^{-4}(t-606)+0.206,606 \leq t<616 \\
5.01 \times 10^{-6}(t-616)+0.213,616 \leq t<2066 \\
0.22 \exp [0.012(t-2066)], 2066 \leq t<2105
\end{array}\right.
$$

The time period between two recovery stages is for the decision-making process. After the unloading, the performance was found to be stable but at a low level. The recovery measure was then stopped for an overall assessment. It was until 3 years later that the metro company decided to speed up the recovery by soil grouting. During the stop between the two recovery stages, the performance evolution function $s(t)$ can be represented by Eq. 8 b.

\subsection{Metric of resilience}

According to Fig. 3, the real performance transition with time duration for the tunnel lining subjected to the extreme surcharge case in this paper is plotted in Fig. 10. The hollow dots represents the real data, while the solid line stands for the real performance evolution functions, e.g., response curve $f(t)$ (Eq. 7) and recovery curve $r(t)$ (Eq. 8). The dash line is the normal performance $Q(t)$. The resilience index is the ratio of the green area over the total area consisting of the green one and the red one, shown in Fig. 10. The red area stands for the loss of the total resilience during the time from Dec 2008 to Jul 2014. 


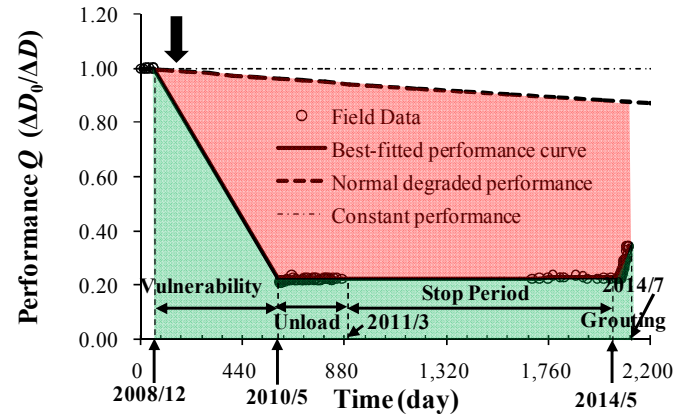

Fig. 10. Transition of performance status against time duration subjected to surcharge loading

Table 2. Resilience metric and status parameter

\begin{tabular}{ll}
\hline Status parameter & Ring No. 388 \\
\hline Performance once the surcharge loaded $Q_{\mathrm{i}}$ & 0.995 \\
Performance response to surcharge $Q_{\mathrm{f}}$ & 0.206 \\
Recovered performance $Q_{\mathrm{r}}$ & 0.343 \\
Average robustness factor $F$ & 0.616 \\
Decision time factor $S$ & 0.239 \\
Average recovery factor $R$ & 0.236 \\
Resilience index Re & 0.341 \\
Loss of resilience 1-Re & $66 \%$ \\
\hline
\end{tabular}

Table 2 shows the calculated numeric metric of resilience by using Eq. 2. The averaged performance response factor $F$ is 0.616 , and the averaged recovery factor $R$ is 0.236 . The factor $S$ considering the recovery stop is 0.239 . The resilience index $R e$ is equal to 0.34 , which means $66 \%$ of the structural resilience has been lost due to the extreme surcharge.

\section{DISCUSSION}

From the above case, it is clear that the residual resilience of the structural performance is relatively small, i.e., more than three quarters of the total resilience has been lost. This result could be attributed into two reasons:

1) The discovery of the surcharge loads is too late to respond. Almost 550 days has elapsed until the discovery. Suppose that the surcharge was discovered 11 days later from Dec 2008, then the $2 \%$ loss of the performance calculated from Eq. 7 will be fully recovered back only by the unloading of the surcharge (Eq. 8a). Hence, in order to monitoring the structural response in real time, some of the smart monitoring and inspection technique is greatly helpful in this sense.

2) The stop between two recovery stages is too long, which lost the rapidity dimension for a resilient structure. Almost 3 years has passed after the unloading of the surcharge and before the soil grouting. The weight of the factor $S$ in Eq. 2a, i.e., $\left(t_{\mathrm{s}}-t_{\mathrm{f}}\right) / \Delta T=0.59$, is the most significant weight in calculating the resilience index. A resilient structure is not only referred to the recovery ability, i.e., $Q_{\mathrm{r}}$, but also referred to the rapidity
$\Delta T$.

It should be noted that the subsequent performance $Q(t)$ after the soil grouting might experience a further decrease due to the long-term effect of the materials, such as grouting and concrete, etc. It is out of the scope of this paper that focuses on the structural resilience during the response and recovery periods.

\section{CONCLUSION}

A detailed framework for the assessment of the tunnel resilience in terms of the performance of horizontal convergence is presented in this paper. The framework is illustrated by using a field case study. Some concluding remarks can be drawn as follows:

1) By applying to the field case, the detailed frame of the resilience metric is found to be reasonable and applicable for the resilience assessment for the lifetime performance of tunnel linings. The tunnel convergence is selected as the performance index, while the ratio of the robustness area over the total area is defined as the resilience index.

2) In this case, the performance of the convergence can be recovered by $16 \%$ of its performance with the help of two recovery measures, but the loss of the resilience is still large. The reason is due to the loss of rapidity both in the response period and also in the recovery period.

3) The smart structural health monitoring (SSHM) and inspection techniques would be greatly helpful to monitoring the structural response in real time and, on the other hand, will increase the structural resilience to ensure the safety of the structures and lives.

\section{ACKNOWLEDGEMENTS}

This study is substantially supported by the National Basic Research Program of China (2011CB013800), Natural Science Foundation Committee program (51278381). Hereby, the authors are grateful to these programs. Besides, the field data are collected with the help of Mr. Shao Hua from Shanghai Shentong Metro Company, Ltd. The authors are also grateful to him.

\section{REFERENCES}

1) Ayyub, B. M. (2014): Systems Resilience for Multihazard Environments: Definition, Metrics, and Valuation for Decision Making. Risk Anal., 34(2), 340-355.

2) Francis, R., and Bekera, B. (2014): A metric and frameworks for resilience analysis of engineered and infrastructure systems. Reliab. Eng. Syst. Saf., 121, 90-103.

3) Karen, I. S. (2014): Resilience-an emerging paradigm of danger or of hope? Disaster Prevention and Management, 23(1), 67-80.

4) Mair, R. J. (2008): Tunnelling and geotechnics: new horizons. Geotechnique, 58(9), 695-736.

5) Ministry of Transportation of the People's Republic of China (MTPRC) (2004): Code for design of road tunnel. China Comunication Press, Beijing. 